\title{
Dental pulp stem cells: function, isolation and applications in regenerative medicine
}

\author{
Marco Tatullo $^{1}$, Massimo Marrelli ${ }^{2}$, Kevin M. Shakesheff ${ }^{3 *}$ and Lisa J. White ${ }^{3}$ \\ ${ }^{1}$ Tecnologica Research Institute, Regenerative Medicine Section, St. E. Fermi, Crotone, Italy \\ ${ }^{2}$ Calabrodental, Maxillofacial Unit, St. E. Fermi, Crotone, Italy \\ ${ }^{3}$ Wolfson Centre for Stem Cells, Tissue Engineering and Modelling (STEM), School of Pharmacy, University of Nottingham, UK
}

\begin{abstract}
Dental pulp stem cells (DPSCs) are a promising source of cells for numerous and varied regenerative medicine applications. Their natural function in the production of odontoblasts to create reparative dentin support applications in dentistry in the regeneration of tooth structures. However, they are also being investigated for the repair of tissues outside of the tooth. The ease of isolation of DPSCs from discarded or removed teeth offers a promising source of autologous cells, and their similarities with bone marrow stromal cells (BMSCs) suggest applications in musculoskeletal regenerative medicine. DPSCs are derived from the neural crest and, therefore, have a different developmental origin to BMSCs. These differences from BMSCs in origin and phenotype are being exploited in neurological and other applications. This review briefly highlights the source and functions of DPSCs and then focuses on in vivo applications across the breadth of regenerative medicine. (C) 2014 The Authors. Journal of Tissue Engineering and Regenerative Medicine published by John Wiley \& Sons, Ltd.
\end{abstract}

Received 6 August 2013; Revised 16 December 2013; Accepted 17 March 2014

Keywords dental pulp stem cells; pulp regeneration; bone regeneration; neural regeneration; angiogenesis; isolation

\section{Physiology of dental pulp and cell function}

Tooth development occurs through inductive signalling between oral epithelial and ectomesenchymal cells, both originating from migrating neural crest cells. These interactions create an outer layer of enamel, formed by epithelial cell-derived ameloblasts (Shi et al., 2005). Enamel covers the tooth crown, exposed in the oral cavity and cementum, a thin layer of bone-like tissue covers the tooth root surface. Underlying these hard tissues is an inner layer of vital mineralized dentin, synthesized by odontoblasts, and finally the pulp core. The dental pulp contains connective tissue, mesenchymal cells, neural fibres, blood vessels and lymphatics (Liu et al., 2006) and its main functions are to produce dentin and maintain the biological and physiological vitality of the dentin. Blood vessels

*Correspondence to: Kevin M. Shakesheff, Wolfson Centre for Stem Cells, Tissue Engineering and Modelling (STEM), School of Pharmacy, University of Nottingham, UK. E-mail: kevin. shakesheff@nottingham.ac.uk and nerve bundles enter the dental pulp through the apical foramen and provide nutrition and a responsive sensory nervous system.

This complex structure provides rigidity and stability; however, teeth remain vulnerable to damage caused by mechanical trauma, chemicals, cancer and bacterial infections. Unlike bone, which can remodel and repair, teeth do not readily undergo complete regeneration and demonstrate limited reparative processes (Shi et al., 2005). After the crown is formed, ameloblasts undergo programmed cell death and lose the ability to repair enamel in vivo. Although odontoblasts cannot repair dentin in vivo, their progenitor or stem cells are capable of migrating into the dentin surface and differentiating into odontoblasts to form reparative dentin. Unlike primary dentin, this reparative dentin is poorly organized, with irregular dentinal tubules embedded in the dentin matrix, but it provides a protective barrier to the dental pulp (Liu et al., 2006).

The ability to create new odontoblasts throughout life in response to damage suggested a source of stem cells within the dental pulp. Gronthos et al. (2000) isolated and characterized these stem cells and termed them dental pulp stem cells (DPSCs). These cells showed similarities, 
and important differences, to human bone marrow stromal cells (BMSCs). Subsequently, Shi and Gronthos (2003) demonstrated that a majority of DPSCs expressed the pericyte marker $3 G 5$ compared to a minority expression in BMSCs. Recently, Karaöz et al. (2011) extended the comparison of DPSCs and BMSCs [also defined as bone marrow-derived mesenchymal stem cells (BMMSCs)] to confirm intrinsic neuroglial characteristics of the DPSCs and differentiation into both neural and vascular endothelial cells in vitro. Mitsiadis et al. (2011) have reviewed the niche around DPSCs and explored the importance of Notch signalling in the dentin repair process.

\section{Isolation of dental pulp stem cells}

The original report of isolation of human DPSCs used impacted third molars as the tissue source (Gronthos et al., 2000). The pulp was physically removed from the crown and root and subjected to a collagenase and dispase digest. Single cells in suspension were then generated by physical straining of the digested tissue. Colony-forming cells were derived at a high frequency from dental pulp (22-70 colonies $/ 10^{4}$ cells plated) compared with the incidence when stromal cells were isolated from bone marrow. Kawashima (2012) has published a comprehensive review of candidate markers of DPSCs and discussed their neural crest origin; these markers include STRO-1, CD29, CD44, CD73, CD90, CD105, CD146, CD166 and CD271.

A large body of literature is building that explores alternative and improved methods of DPSC isolation and expansion. Hilkens et al. (2013) compared two methods of isolation of DPSCs from impacted third molars. They confirmed no significant differences in a range of marker expressions and differentiation potentials for enzymatic digestion versus cell outgrowth from intact tissue.

The type of tooth to be used in isolation has been intensely investigated. Impacted third molars were used in the original studies reported above, but exfoliated deciduous ('baby') teeth could be an excellent source of cells for banking (Kawashima, 2012). Stem cells derived from exfoliated deciduous teeth are known as SHEDs. The importance of precisely naming cells based on source is emphasized by Kerkis and Caplan (2012), who reported that three populations of cells could be isolated from deciduous tooth pulp. Govindasamy et al. (2012) compared DPSCs isolation from permanent and extracted deciduous teeth. They reported higher proliferation rates in the cells from deciduous teeth (termed SCDs, to differentiate the name from cells derived from exfoliated teeth) compared to cells from permanent teeth. In addition, numerous pluripotent markers were higher, as were neuroectodermal markers. Huang et al. (2008a) reported DPSCs isolation from supernumerary teeth.

As DPSC technologies head towards clinical use, there is a need to modify culture and expansion processes. For example, the use of bovine serum can be problematic in clinical cell therapy. Ferro et al. (2012) have shown that bovine serum can be replaced by a chemically defined medium containing low percentages of human serum. The human serum could be derived from autologous or heterologous sources.

A potential problem with the use of DPSCs is the small number of cells available for isolation, due to the small size of the pulp (especially for exfoliated deciduous teeth). Generating sufficient cells for clinical application would require multiple passages that might reduce the potency of the cultured cells. Lizier et al. (2012) demonstrated that DPSCs could be cultured for 6 months without changes in morphology or the expression of stem cell markers. This raises the prospect that these cells can be expanded to high numbers and used in multiple regenerative medicine applications.

A final issue to consider in the isolation and expansion of DPSCs for clinical use is the ability to cryopreserve teeth prior to isolation. Cryopreservation would allow the banking of teeth in anticipation of the need for DPSCs at some time in the future (Woods et al., 2009; Zhang et al., 2006) and Lee et al. (2010) reported no changes in cell viability and stem cell markers as a result of cryopreservation using controlled cooling rates but no cryopreservant.

\section{Regenerative medicine applications of dental pulp cells}

\subsection{Dental pulp regeneration}

\subsubsection{Early studies}

Maintenance of dental pulp function is critical for the homeostasis of teeth; loss of dental pulp is often followed by tooth fracture and/or periapical disease and, finally, loss of teeth. When dental pulp is infected it is difficult for the immune system to eradicate the infection, due to lack of blood supply to the pulp. Partially removing the infected pulp, termed partial pulpectomy, has proved to be ineffective, as infecting organizms may be left behind (Huang et al., 2009a, 2009b). Thus infection of adult pulp by trauma or caries often necessitates root canal therapy, in which the entire pulp is removed and the pulp cavity disinfected and filled with an artificial material. Biological alternatives to root canal therapy have inspired regenerative endodontics, whereby the diseased or necrotic pulp tissues are removed and replaced with regenerated pulp tissue, capable of revitalizing teeth (Sun et al., 2011). For recent reviews of dental pulp regeneration, the reader is referred to a number of excellent papers (Sloan and Smith, 2007; Sun et al., 2011; Huang, 2011; Nakashima and Iohara, 2011).

Whilst the volume of mature pulp tissue is very small (ca. 10-100 $\mu \mathrm{l}$ ) it is a difficult task to engineer and regenerate this tissue, due to its anatomical location, unique microstructure with different cell types and complex innervations, specific location of dentin and the highly organized structure of dentinal tubules (Huang et al., 2009a, 2009b). 
Although dental pulp tissue engineering was investigated in the late 1990s (Mooney et al., 1996; Bohl et al., 1998), it was the identification of dental pulp stem cells capable of generating dentin that rendered dentin pulp regeneration possible (Gronthos et al., 2000).

Human DPSCs were transplanted in conjunction with hydroxyapatite/tricalcium phosphate (HA/TCP) powder into immunocompromised mice. After 6 weeks DPSCs generated a dentin-like structure lining the surfaces of the HA/TCP particles, comprised of a highly ordered collagenous matrix deposited perpendicular to the odontoblastlike layer (Gronthos et al., 2000). The aligned odontoblast-like cells expressed the dentin-specific protein DSPP and extended as tubular structures within newly generated dentin. The collagen matrix mimicked the structure of primary dentin with ordered perpendicular fibres, rather than reparative dentin, which usually consists of a disorganized matrix. In addition, the DPSC transplants contained a fibrous tissue containing blood vessels, similar to the arrangement found in the dentin-pulp complex in normal human teeth. To assess the self-renewal characteristics of DPSCs, Gronthos et al. (2002) re-isolated stromallike cells from the 3 month-old primary DPSC transplants. After in vitro expansion, human cells were re-transplanted into immunocompromised mice. These secondary transplants produced human alu-positive odontoblasts within a dentin-pulp-like complex containing organized collagen fibres, thus showing that the human DPSCs were able to self-renew in vivo.

In these early studies, transplantation of expanded DPSCs formed a dentin-pulp complex and transplantation of expanded bone marrow mesenchymal stem cells (BMMSCs) formed ectopic bone. The tissue regeneration capability of BMMSCs and DPSCs was further examined by transplantation using human dentin as a carrier (Batouli et al., 2003). Although BMMSCs failed to form mineralized tissue on the surface of dentin or a pulp-like connective tissue, DPSCs generated a reparative dentin-like structure directly on the surface of human dentin, indicating the possibility of using DPSCs in tooth repair.

This isolation and characterization of dental pulp stem cells, combined with increased understanding of tooth development, has led to two major strategies in tooth tissue engineering: in vivo transplantation of stem cells and in vitro culture of stem cells on biodegradable scaffolds and subsequent transplantation in vivo (Galler et al., 2011). Both strategies have found application in pulp regeneration utilizing DPSCs.

\subsubsection{In situ pulp regeneration}

A number of studies have indicated that the DPSCs may be used to regenerate partially lost pulp and dentin. Nakashima's group were able to demonstrate partial regeneration of pulp using porcine pulp cells, cultured as a three-dimensional (3D) pellet (Iohara et al., 2004). The expression of dentin sialophosphoprotein (DSPP) confirmed the differentiation of DPSCs into odontoblasts. Additionally, autogenous transplantation of a bone morphogenetic protein-2 (BMP-2) treated pellet culture onto the amputated pulp of a dog stimulated reparative dentin formation. Similar results were achieved with a 3D pellet culture system of pulp cells electrotransfected with growth/differentiation factor 11 (Gdf11) (Nakashima and Akamine, 2005).

Iohara et al. (2006) continued their investigations of dental pulp regeneration by isolating a side population (SP) of cells from dental pulp based on the efflux of fluorescent dye Hoechst 33342. These SP cells, derived from porcine dental pulp, differentiated into odontoblasts in response to BMP-2. Furthermore, autogenous transplantation of BMP-2-treated canine SP cells induced osteodentin formation in surgically created defects on amputated canine dental pulp Two further fractions of SP cells were isolated from canine dental pulp: $\mathrm{CD}^{-}{ }^{-}$/ $\mathrm{CD}_{146^{-}}$and $\mathrm{CD} 31^{+} / \mathrm{CD} 146^{+}$SP cells were separately cultured as pellets with collagen type I and collagen type III and autogenously transplanted into amputated pulps (Iohara et al., 2009). Pulp-derived CD $31^{-} / \mathrm{CD} 146^{-}$SP cells induced a strong vasculogenic response; cells differentiated into odontoblasts only at the periphery of dentin and thus produced a physiologically normal regenerated pulp tissue.

Complete pulp regeneration with neurogenesis and vasculogenesis occurred in an adult canine model of pulpectomy with autogenous transplantation of pulp $\mathrm{CD} 105^{+}$SP cells with stromal cell-derived factor-1 (SDF-1) (Iohara et al., 2011). Side population $\mathrm{CD} 105^{+}$cells formed pulp-like tissue by day 14 when transplanted with SDF-1 and induced complete apical closure, whereas transplantation of $\mathrm{CD} 105^{+}$cells alone or SDF-1 alone yielded less pulp. This seminal work by Nakashima's group was the first demonstration of complete in situ pulp regeneration.

A recent study from this group has compared the biological characteristics and regenerative potentials of dental pulp, bone marrow and adipose stem cells taken from the same individual (Ishizaka et al., 2012). In this investigation SP cells were further sub-fractionated into CD31- cells, previously shown to stimulate angiogenesis/ vasculogenesis in vitro and in vivo (Iohara et al., 2008). The differential potentials of pulp regeneration of the three SP fractions were determined using an in vivo model previously described (Huang, 2011), whereby the three CD31- SP populations were injected into porcine tooth root fragments prior to transplantation into immunocompromised mice. Whilst pulp-like tissue was observed after transplantation of all three SP fractions, the total volume of regenerated tissue was significantly higher with the dental pulp SP and the density of vasculature and innervations was also higher (Ishizaka et al., 2012).

Nakashima's group have established a potential cell therapy for pulp regeneration harnessing autologous DPSCs, $\mathrm{CD}^{-} 1^{-}$SP cells or $\mathrm{CD}_{105^{+}}$cells together with SDF-1 in a canine pulpitis model. However, the safety and efficacy of labelling CD $31^{-}$SP cells may be a barrier to clinical translation of this work. To overcome this, Iohara et al. (2013) developed a method to obtain human DPSCs from dental pulp based on good manufacturing practice (GMP) guidelines. Transplantation of GMP DPSCs with granulocyte-colony 
stimulating factor (G-CSF) in a canine pulpectomy model regenerated pulp tissue. G-CSF enhanced pulp regeneration and appeared to create a favourable environment for migration of cells, inhibition of apoptosis, suppression of inflammation and induction of angiogenesis and neurogenesis.

Similar to the approach of Nakashima's group, Zheng et al. (2012) investigated direct pulp capping on the pulp chamber roof in a porcine model. Autologous porcine DPSCs were combined with a $\beta$-tricalcium phosphate ( $\beta$-TCP) scaffold. Regenerated dentin and restoration of the defect was obtained after 16 weeks, demonstrating the capacity of transplantation of DPSCs for in situ dentin regeneration.

\subsubsection{De novo pulp regeneration}

When the entire pulp tissue is lost, regeneration requires the de novo creation of pulp. In order to create functional pulp for clinical application, several issues must be considered: first, regenerated pulp tissue must be vascularized, even through the blood supply occurs only from the apical foramen; second, newly differentiated odontoblasts should form on the existing dentinal wall of the root canal space; and finally, new dentin must be produced by the differentiated odontoblasts on the existing dentin (Huang, 2009; Sun et al., 2011). A number of research groups have attempted to engineer pulp tissue replacements similar in physiology to normal pulp tissue that have the potential to be used in regenerative endodontic procedures.

Prescott et al. (2008) experimented with the tissue engineering triad of stem cells, scaffold and growth factor. They measured the effectiveness of DPSCs in conjunction with a collagen scaffold and dentin matrix protein 1 (DMP1) to form dental pulp-like tissue in a subcutaneous transplantation in mice. Mineral trioxide aggregate, commonly used for perforation repair, was used as the control repair material. Organization of newly derived pulp tissue was only observed in the group containing all triad components. Further to this, Alsanea et al. (2011) utilized the same combination of DPSCs, DMP1 and a collagen scaffold in a simulated perforation model. The presence of DMP1 promoted the differentiation of DPSCs within the collagen scaffold into odontoblast-like cells capable of forming a highly cellular, vascularized matrix. Previous work, however, has raised concerns regarding the use of collagen in pulp regeneration: Huang et al. found that in vitro culture of human DPSCs within collagen gels caused severe gel contraction (Huang et al., 2006).

Material platforms for dental pulp engineering have included other hydrogels, natural and synthetic polymers and bioceramics (Galler et al., 2011). The suitability of different materials has been evaluated by in vitro assessment, e.g. Gebhardt et al. (2009) explored cell survival and degradation within three types of tissue-engineering scaffolds. DPSCs and periodontal ligament stem cells (PLSCs) were seeded on synthetic open cell polylactic acid (polymer) scaffolds, bovine collagen scaffolds and calcium phosphate bioceramic scaffolds, with optimal cell survival occurring in the polymer and collagen scaffolds. In a similar study, Kim et al. (2009) examined the attachment, growth and differentiation of human DPSCs on three naturally occurring scaffolds: collagen, gelatin and chitosan. Whilst DPSCs attached and proliferated rapidly on both gelatin and collagen, a mineralized extracellular matrix was only observed with collagen. Galler et al. (2011) investigated PEGylated fibrin as a suitable scaffold and evaluated the proliferation and differentiation of DPSCs, PLSCs and stem cells from human exfoliated deciduous teeth (SHEDs) in this matrix. Differences in cellular morphology, ECM production and mineral deposition were observed between cell types; DPSCs appeared elongated and fibroblast-like, produced little collagen and showed some increased expression of DSPP and DMP-1. Other researchers have also considered material based in vitro odontogenic differentiation of hDPSCs: Nam et al. (2011) indicated that calcium phosphate $(\mathrm{CaP})$ porous granules may induce odontogenic differentiation and demonstrated upregulation of DSPP and DMP-1, increased secretion of dentin sialoprotein (DSP) and increased mineralization. More recently, Qu and Liu (2013) fabricated nano-fibrous gelatin-silica bioactive glass (NF-gelatin-SBG) hybrid scaffolds that mimicked the nano-structured architecture and chemical composition of natural dental ECM for in vitro differentiation of hDPSCs. Expression of marker genes for odontogenic differentiation (Col1, ALP, OCN, DSPP and DMP-1) was significantly higher in the NF-gelatin-SBG scaffolds compared to the NF-gelatin group, with enhanced ECM secretion and mineral deposition in the SBG hybrid group.

In contrast to in situ pulp regeneration, much of the research surrounding in vivo de novo regeneration has focused on the capacity of DPSCs to form dentin rather than a whole regenerated pulp (Kawashima, 2012). For example, although the Jansen group were able to form well-vascularized DSPP-expressing tissue in a subcutaneous mice model, they questioned the practical utilization of DPSCs. since little hard tissue was formed (Zhang et al., 2006). The Jansen group sought to improve this by utilizing STRO-1-selected DPSCs (Yang et al., 2009a, 2009b). Abundant hard tissue was formed after subcutaneous implantation of rat STRO-1selected DPSCs on a ceramic carrier; the addition of BMP-2 further accelerated odontoblastic differentiation of DPSCs (Yang et al., 2009b). Furthermore, BMP-2 transfection of DPSCs produced a more effective induction toward the odontoblast phenotype and abundant mineralized tissue (Yang et al., 2009a). Yet, despite the upregulation of dentin-specific proteins (DSPP and DMP-1), the hard tissue formed was bone like in appearance and morphology. Mineralized tissue formation also occurred when hDPSCs were cultured with simvastatin or BMP-2 for 7 days and then mixed with HA/TCP power and transplanted subcutaneously into immunocompromised mice (Okamoto et al., 2009). However, simvastatin significantly increased DSPP expression in DPSCs compared to BMP-2.

The Ma group also examined odontogenic differentiation of hDPSCs, with the material platform of nanofibrous (NF)-poly(L-lactic acid) (PLLA) scaffolds in vitro and in vivo (Wang et al., 2010, 2011). Combined treatment of dexamethasone and BMP-7 had a pronounced effect: odontogenic differentiation and hard tissue formation 
increased and the tissue itself was more organized, with collagen and calcium depositions located at the walls of the scaffold pores and pulp-like tissue observed in the middle of the pores. A comparison of differently architectured scaffolds demonstrated that nano-fibrous scaffolds provided a better extracellular microenvironment for odontogenic differentiation of DPSCs compared to solid walled scaffolds, with upregulation of osteocalcin (OCN) and DSPP and increased mineralization (Wang et al., 2011). However, despite the DSP positive staining and hard tissue formation, the tissue formed did not resemble tubular dentin tissue. In contrast to this, El-Blackly et al. (2008) formed osteodentin-like structures as well as tubular bilayered structures of vertically aligned tubules resembling dentin from isolated dental pulp stem cells from rabbit teeth. DPSCs were seeded onto poly(lacticco-glycolic acid) polymer scaffolds and transplanted subcutaneously into rabbits.

Seminal work by Nör's group engineered dental pulp constructs by seeding a PLLA scaffold within a tooth slice model with SHEDs (Cordeiro et al., 2008). The acellular tooth matrix contained endogenous morphogenetic signals and, when transplanted into immunodeficient mice, the resultant tissue presented architecture and cellularity that resembled physiological dental pulp. Following on from this, the Nör group investigated the effect of dentin, present in the tooth slice model, and different scaffold porogens upon the differentiation of hDPSCs into odontoblasts (Demarco et al., 2010), A similar result was obtained with hDPSCs as with SHEDs, whereby the morphogenetic cues from the tooth slice influenced differentiation; additionally, a tissue with morphology resembling normal dental pulp was observed inside the tooth slice/scaffold.

Concurrent to this, Huang et al. (2009a, 2009b) demonstrated de novo synthesis of vascularized human pulp/ dentin-like tissues in an emptied human root canal space, 5-6 mm deep, with an end opening of only $\sim 2.5 \mathrm{~mm}$. Stem cells from apical papilla (SCAP) were combined with DPSCs, seeded onto poly (DL-lactic acid-co-glycolic acid) (PLGA) scaffolds and then inserted into the canal space of root fragments. These tooth constructs were then subcutaneously implanted into severe combined immunodeficient mice (Huang et al., 2010). A pulp-like tissue with well-established vascularity filled the entire root canal space. Additionally, a continuous layer of dentin-like tissue was deposited onto the canal wall and appeared to be produced by a layer of newly formed odontoblast-like cells expressing DSP, alkaline phosphatase (ALP), bone sialoprotein (BSP) and CD105 (Huang et al., 2009a, 2009b).

Recently, Kodonas et al. (2012) utilized a similar root fragment model and seeded porcine DPSCs onto collagen or PLGA scaffolds prior to immersing the cells/scaffold into root canals. These constructs were then implanted into the jawbones of adult minipigs. After 10 weeks, newly formed organic matrix of tubular pre-dentin form was deposited on the root canal walls and columnar polarized cells were adjacent to this matrix. Although the large opening ( $>2 \mathrm{~mm}$ ) of the canal wall was sufficient for vascularization in the study by Huang et al. (2010), in this work necrotic tissue was observed at the central area of some implants (Kodonas et al., 2012). This underscores the importance of vascularization in regenerated pulp tissue, since the blood supply can only come from the apical foramen.

The survival of rat DPSCs in a vascularized chamber was investigated by Thompson's group, who adapted an arteriovenous loop (AVL) and vascular pedicle tissueengineering chamber model in order to study dental pulp tissue generation in vivo (Srisuwan et al., 2012a). DPSCs were mixed with collagen scaffolds, with or without slow-release bone morphogenetic protein 4 (BMP-4) gelatin microparticles and fibroblast growth factor 2 (FGF2); constructs were transplanted into a vascularized chamber in the rat groin. Signalling molecules were provided by use of either a dentin cylinder, to mimic the rigid structure of a tooth, or by dentin fragments incorporated into the collagen matrix inside the chamber. The use of dentin fragments produced healthy vascularized tissue after 8 weeks, with surviving DPSCs observed around the dentin fragments. Further to this, Srisuwan et al. (2012a) utilized a collagen 1 hydrogel scaffold with gelatin microparticles conjugated with FGF2, vascular endothelial growth factor (VEGF) and platelet-derived growth factor (PDGF) and rat DPSCs. A pulpless tooth chamber was filled with the collagen/gelatin/DPSCs combination and either placed into a hole created in the femoral cortex or into its own socket, respectively (Srisuwan et al., 2012a). Interestingly, tooth revascularization and tissue generation only occurred in the allogeneic femur group, demonstrating the importance of a direct vascular supply.

Whilst the vascularization studies (Srisuwan et al., 2012a, 2012b) provide important lessons for tissue generation, it is the work of Nör and Huang's groups (Cordeiro et al., 2008; Demarco et al., 2010; Huang et al., 2010) that most clearly indicate the path required for de novo regeneration: molecular cues from a dentin environment, whether it be a tooth slice model, root fragment or root canal, are essential for the creation of physiologically relevant dentin-pulp tissue (Cordeiro et al., 2008; Huang et al., 2009a, 2009b; Demarco et al., 2010). This has recently been demonstrated by the odontogenic differentiation of hDPSCs and periodontal ligament cells when cultured on a decellularized extracellular matrix scaffold (Ravindran et al., 2014). DPSCs were cultured in a collagen/chitosan hydrogel for 2 weeks; the cells were then lysed and DNA digested to produce an ECM-embedded collagen/chitosan scaffold. Characterization of the ECM scaffold showed that it contained a rich source of matrix proteins, matrix metalloproteases and growth factors, capable of delivering molecular cues. Subcutaneous implantation of these ECM scaffolds containing DPSCs showed the formation of dental pulp-like tissue with cells expressing DSP and dentin phosphophoryn (DPP).

\subsection{Tooth reconstruction}

As our understanding of tooth formation, the stem cell niche and regenerative mechanisms extends, it may be 
possible to generate a method to biologically replace lost teeth. A functional biological replacement tooth must include generation of a root and periodontal ligament with nerve and blood supplies. The crown, although the most visible part of the tooth, is actually less important in terms of regeneration, since replacement of crowns with synthetic functioning options is possible (Volponi et al., 2010).

Mammalian teeth develop by a series of interactions between embryonic oral epithelial cells and neural crestderived mesenchyme, with the epithelium providing the initial instructional signals to the mesenchyme at the beginning of tooth development. (Tucker and Sharpe, 2004; Zhang et al., 2005). In order to bioengineer a tooth from non-embryonic cells, one of the cell populations, either epithelial or mesenchymal, must be able to provide the inductive signals to the other. In vitro embryonic tooth primordia have been generated from adult stem cells combined with instructive embryonic oral epithelium (Ohazama et al., 2004), adult epithelial cells combined with embryonic tooth inducing mesenchyme (Angelova Volponi et al., 2013) or by the development of tooth germ models utilizing harvested cells from tooth buds combined with materials (Zhang et al., 2009). Both the Sharpe (Ohazama et al., 2004) and Tsuji (Ikeda et al., 2009) groups have demonstrated that embryonic tooth primordia can develop into complete teeth, following transplantation in the adult oral cavity, and after sufficient time will form roots and erupt.

Since adult DPSCs are derived from neural crest, they are a potential replacement for embryonic mesenchyme in the generation of tooth primordia; however, this application has not yet been demonstrated. Hung et al. (2011) were able to utilize DPSCs to form tooth-like structures in rabbit alveolar sockets but there was no visible tooth eruption in any of the graft sites. Yu et al. (2012) demonstrated that DPSCs pellet reassociated with adult rat apical bud cells formed crown-like structures in vivo containing distinct regions of enamel, dentin, pre-dentin and both ameloblast and odontoblast layers. Until recently, no root-like structures had been produced using DPSC-based regeneration (Yan et al., 2010a); the latter authors have postulated that the future lies in using iPS cells from autologous DPSCs isolated from a patient's own dental pulp. The iPS cells could generate dental epithelial cells, with embryonic-like properties, that could be recombined with the autologous DPCSs and transplanted to form a tooth. For biological tooth formation to be a viable alternative to dental implants, adult sources of human epithelial and mesenchymal cells must be indentified and obtained in sufficient quantities (Angelova Volponi et al., 2013). The use of induced pluripotent stem cells (iPS cells) from DPSCs may indeed provide a future solution to this problem.

In the interim, regeneration of mineralized tooth roots has become a practical goal. Previously, a tooth root-like structure with periodontal-ligament (PDL) like tissue was formed in a HA/TCP cylinder loaded with stem cells from apical papilla (SCAP) and PDL stem/progenitor cells
(Sonoyama et al., 2006). A recent study, however, has shown the utility of DPSCs to produce putative dentin/ cementum, PDL and alveolar bone complex (Lee et al., 2014). 3D layer-by-layer fabrication of a multiphase scaffold enabled precise control of microarchitecture in different regions in conjunction with spatiotemporal delivery of three recombinant human proteins. DPSCs were stimulated by bioactive cues and produced type I collagen fibres that inserted into a $\mathrm{DSPP}^{+}$mineralized matrix on one side and a $\mathrm{BSP}^{+}$bone-like tissue on another side (Lee et al., 2014).

\subsection{Bone tissue engineering}

Dental stem cells are a potential source for bone tissue engineering. A new c-kit ${ }^{+} / \mathrm{CD} 34^{+}$population of DPSCs were selected from dental pulp, expanded and cultured (Laino et al., 2005). This stem cell population, referred to as stromal bone producing DPSCs (SBP/DPSCs), differentiated into $\mathrm{CD}_{4} 4^{+} / \mathrm{Runx}^{+}$osteoblast precursors and subsequently into osteoblasts with the addition of $20 \%$ fetal bovine serum (FBS). In vitro osteogenic culture for 50 days produced a fibrous bone-like tissue composed of a densely mineralized matrix. In vivo transplantation of DPSCs into immunocompromised rats generated fibrous lamellar bone tissues containing osteocytes.

The osteogenic differentiation capacity of DPSCS has been well demonstrated in vitro and in vivo, with strong alkaline phosphatase results (Lindroos et al., 2008) and expression of bone-specific markers within newly formed bone (d'Aquino et al., 2007, 2008). The osteogenic capacity of DPSCs has led to their use in combination with 3D polymeric scaffolds to produce bone-like hard tissues. In attempting to engineer de novo pulp regeneration, wellmineralized hard tissue, with distinct concentric lamellae and partially developed bone marrow-like haematopoietic tissue, was formed during subcutaneous implantation of rat STRO-1-selected DPSCs with a 3D porous HA/TCP carrier (Yang et al., 2009b). Both the addition (Yang et al., 2009b) and transfection (Yang et al., 2009a) of BMP-2 enhanced the production of mineralized tissue. A similar effect was observed by Ikeda et al. (2010), whereby in vitro culture of DPSCs with recombinant human BMP-2 (rhBMP-2) caused prominent osteo-inducibility of DPSCs, which in turn generated abundant bone-like tissues in vivo.

Osteogenic differentiation of DPSCs has occurred in conjunction with other material platforms: DPSCs were cultured in vitro on different titanium surfaces and differentiated into osteoblasts, and produced bone on lasersintered surfaces (Mangano et al., 2010). More recently, DPSCs were pre-differentiated into osteoblast-like cells and seeded onto collagen-hydroxyapatite-poly(L-lactideco- $\varepsilon$-caprolactone) (Col1-HA-PLCL) composite scaffolds (Akkouch et al., 2014). The composite scaffold promoted adhesion, proliferation and differentiation of the osteoblastlike cells, with ECM mineralization occurring throughout the scaffold. More recently, Kanafi et al. (2013b) investigated the feasibility of immobilizing DPSCs within alginate 
microspheres and investigated the osteogenic differentiation potential in vitro. Enhanced mineralization, protein secretion and an upregulated osteo-related gene profile resulted from immobilization and, interestingly, immobilization triggered osteogenic differentiation of DPSCs without the use of induction factors in the medium.

There have been several promising in vivo studies of bone repair using DPSCs. Building on from their work revealing the capability of DPSCs to form woven bone in vitro (Laino et al., 2006) and well-vascularized lamellar bone in vivo (d'Aquino et al., 2007), Papaccio's group demonstrated that autologous DPSCs seeded onto collagen sponge scaffolds could be used to repair alveolar defects (d'Aquino et al., 2009). The autografts repaired alveolar bone defects of the mandible produced after extraction of impacted third molars; the quality and quantity of bone regeneration was optimal, resulting in an effective therapeutic strategy.

Yamada et al. (2010) investigated canine DPSCs within an osseointegration study. This served as a model for bone augmentation that may be required in atrophic areas prior to receiving dental implants for the restoration of normal mastication. Further to this, DPSCs were implanted with platelet-rich plasma (PRP) into bone defects in the canine mandible (Yamada et al., 2011). Histological observations showed well-formed mature bone, positive for osteocalcin and containing neovascularization. These studies further indicate the potential of DPSCs for bone regeneration in oral maxillofacial surgery and craniofacial anomalies.

Recent work has also provided the potential utility of DPSCs in regeneration of critical-sized bone defects. Rat DPSCs were utilized in a rat calvarial critical defect model in conjunction with a granular deproteinized bovine bone (GDPB) or $\beta$-TCP scaffold (Annibali et al., 2014). In this preliminary data, a tendency to increase the bone mineral density was observed when DPSCs were implanted in the GDPB scaffold. Maraldi et al. (2013) also utilized DPSCs in a rat calvarial critical-sized defect model. Human DPSC seeded collagen sponges showed almost complete bridging of the defect by 8 weeks.

Regulation of DPSC differentiation is crucial for clinical use in cell therapies and regenerative medicine. The topographical design of biomaterials may be optimized to achieve this. The interaction of surface topographical parameters upon attachment, morphology, proliferation and osteogenic differentiation of DPSCs was recently undertaken (Kolind et al., 2014), with alterations in pillar topography resulting in enhanced mineralization.

\subsection{Other applications in cell therapies}

\subsubsection{Neurology}

Due to the low incidence of adult neural stem cells (NSCs) and issues with harvesting, the use of other stem cell types with neural potential may be required to achieve neuroregeneration. Although BMMSCs have been assessed as candidates for neuronal therapy (Lindvall et al., 2004), efficiency of differentiation and accessibility may limit their use. Stem cells isolated from dental pulp may be an alternative, since they show expression of embryonic stem cell (ESC) pluripotency markers, such as Oct-4, Nanog, SSEA-4 and TRA-1-60 (Kerkis et al., 2006), and also display multipotency markers indicating chondrogenic and osteogenic tissue formation and spontaneous neural differentiation (Kim et al., 2012). Research into the generation of neural lineage cells from dental stem cells has shown that DPSCs express various neural markers upon stimulation with neural differentiation medium; many studies have been performed to establish effective culture strategies to promote neurosphere formation and neural induction (Nosrat et al., 2004; Takeyasu et al., 2006; Govindasamy et al., 2010; Janebodin et al., 2011; Király et al., 2011; Karaoz et al., 2011). A comprehensive examination of culture strategies was recently reported (Young et al., 2013). The molecular regulation of DPSC differentiation has also been investigated by Ge et al. (2013), who examined the role of the distal c-terminus of voltage-gated L-type $\mathrm{Ca}^{2+}$ channel in orientating DPSCs differentiation toward the neuronal phenotype.

In addition to studies of in vitro differentiation, researchers have attempted to regenerate nerve tissue utilizing transplantation of DPSCs. Arthur et al. (2008) postulated that adult stem cell populations derived from the neural crest would possess a predisposition for neuronal differentiation and repair. Ex vivo expanded hDPSCs responded to neuronal induction both in vitro and in vivo, acquired a neuronal morphology and expressed gene and protein neuronal-specific markers. In addition, given appropriate environmental cues, DPSCs influenced endogenous recruitment of neural stem cells (Huang et al., 2008b) and generated neurospheres (Sasaki et al., 2008a). Further to this, Arthur et al. (2009) investigated stem cell-mediated neuroplasticity in a receptive host nervous system. An avian embryonic model system was adapted to investigate axon guidance in vivo after transplantation of hDPSCs. Transplanted hDPSCs altered patterns of axonal migration and may also assist in the homing of endogenous neural stem cells to the site of transplantation.

Although undertaken with dental pulp cells (DPCs) rather than DPSCs, seminal work by Nosrat et al. (2001) demonstrated that dental pulp-derived cell grafting promoted survival of injured motor neurons in a rat model of spinal cord injury. In addition, DPCs from both rats and humans produced and secreted neurotrophic factors, including nerve growth factor (NGF), brain-derived neurotrophic factor (BDNF) and glial cell line-derived neurotrophic factor (GDNF) (Nosrat et al., 2004) and these promoted the survival of sensory and domaminergic neurons. In a separate study, DPCs exerted a neuroprotective effect in in vitro models of Alzheimer's and Parkinson's disease (Apel et al., 2009). The ability to produce and secrete growth factors is of prime importance, since these factors may induce the differentiation of endogenous cell types into those cells required at the place of injury or elicit secretion of other neurotrophic factors from endogenous cells to improve 
tissue regeneration (Martens et al., 2013). This was observed in a study by de Almeida et al. (2011), whereby transplantation of human dental pulp cells (hDPCs) into the centre of a spinal cord lesion in mice produced larger areas of white matter preservation and better tissue organization. It was postulated that the secretion of neurotrophic factors may have led to stimulation of collateral sprouting, which can enhance functional outcomes. Correspondingly positive results were obtained with transplantation of hDPSCs into a rat model of spinal cord injury (Sakai et al., 2012). A recent review of applications of DPSCS and SHEDs in spinal cord injury models has shown that the microenvironment of transplanted stem cells affects their capacity for differentiation; an injured spinal cord contains high levels of pro-inflammatory mediators which may activate the oligodendrocyte-specific differentiation cascade (Yamamoto et al., 2014).

Dental pulp cells have also been proposed as a treatment for peripheral nerve injury (Sasaki et al., 2008b, 2011). Initially, dental pulp cells were transplanted into collagen gels and infused within a silicon tube, which was positioned within a $7 \mathrm{~mm}$ gap in the buccal branch of rat facial nerve. The dental pulp cells formed blood vessels and myelinating tissue and contributed to the promotion of normal nerve regeneration. In a subsequent study, a degradable poly(DL-lactide-co-glycolide) (PLGA) tube was developed to avoid the need for a potential second operation to remove the silicon tube. The PLGA tubes were resorbed in vivo and nerve regeneration was observed 2 months after the transplantation. Although this work was undertaken with dental pulp cells as a whole, rather than DPSCs, it illustrates the potential of DPCSs in neuronal regeneration.

A recent study assessed the therapeutic benefit of implanted rat DPSCs into the vitreous body of the eye after a surgically induced optic nerve crush injury (Mead et al., 2013); DPSCs promoted neurotrophic-mediated survival of rat ganglion cells and axon regeneration.

In addition to central nervous system (CNS), optic and peripheral nerve injuries, stem cell therapy has been proposed for treatment of stroke. In stroke, occlusion of a cerebral artery leads to ischaemia in a restricted region of the CNS (Nakashima et al., 2009). It has previously been shown that transplanting differentiated neural stem cells isolated from dental pulp improved motor disability and reduced infarct volume (Yang et al., 2012). Therapeutic translation of DPSCs to stroke treatment has recently been tested in a rodent cerebral ischaemic model (Nakashima et al., 2009; Sugiyama et al., 2011; Leong et al., 2012). Transplantation of porcine CD31-/CD146 SP cells accelerated neovascularization of the ischaemic zone and enhanced neuronal regeneration (Nakashima et al., 2009). In a further study, transplanted porcine $\mathrm{CD}^{-} 1^{-} / \mathrm{CD} 146^{-}$SP cells migrated to the peri-infarct area, released neurotrophic factors and promoted migration and differentiation of neural progenitor cells in the subventricular zone (Sugiyama et al., 2011).

Similarly, intracerebral transplantation of hDPSCs $24 \mathrm{~h}$ after focal cerebral ischaemia in a rodent model resulted in significant improvement in forelimb sensorimotor function. Improvements to function appeared to be mediated by DPSC-dependent paracrine effects (Leong et al., 2012), similar to those observed in the treatment of spinal cord injuries with hDPCs (de Almeida et al., 2011). This study provides preclinical evidence of the unique therapeutic potential of hDPSCs to stimulate and modulate local repair response.

\subsubsection{Angiogenesis and vasculogenesis}

Stem cells and endothelial progenitor cells (EPCs) can be utilized to stimulate vasculogenesis as a potential treatment for ischaemic disease (Isner et al., 1999). Correspondingly therapeutic benefits of injection of bone marrow- or adipose-derived MSCs after myocardial infarction (MI) and other heart diseases have also been reported (Dai et al., 2005, Wollert and Drexler 2005). Several studies have investigated the potential of DPSCs and sub-fractions of DPSCs as modes of treatment for MI and ischaemia (Gandia et al., 2008; Iohara et al., 2008; Nakashima et al., 2009).

Gandia et al. (2008) investigated the potential of hDPSCs as treatment of induced MI in a rat model. Human DPSCs were able to repair the infarcted myocardium, with an observed increase in number of vessels and reduction of infarct size; this was likely due to secretion of paracrine factors, such as VEGF.

A highly vasculogenic side population of cells was isolated from porcine dental pulp (Iohara et al., 2008). The CD31 ${ }^{-} /$CD146 ${ }^{-}$SP expressed CD34 and VEGFR2 at similar levels to that expressed by EPCs and formed extensive networks of cords and tube-like structures on Matrigel, demonstrating neovascularization ability. Local transplantation of this subfraction in a mouse hind-limb ischaemia model resulted in successful engraftment and increase in blood flow including high density of capillary formation. Similar successful engraftment was achieved with $\mathrm{CD}_{105}{ }^{+}$SP cells (Nakashima et al., 2009). More recently, Bronckaers et al. (2013) demonstrated that hDPSCs induced paracrine-mediated angiogenesis. Human DPSCs produced high amounts of angiogenic molecules, stimulated endothelial cell migration by activation of the P131AKT and MEK-ERK pathways and significantly induced the formation of blood vessels in a chicken chorioallantoic membrane model. This study provides evidence of the suitability of DPSCs for treatment of pathologies correlated with inadequate angiogenesis such as stroke and myocardial infarction.

\subsubsection{Endocrinology}

Cell therapy treatments for liver disease require effective stem-cell derived hepatocytes. DPSCs have been differentiated to produce hepatocyte-like cells (HLCs) (Ishkitiev et al., 2012) with acquired hepatocyte functions, such as glycogen storage and urea production (Patil et al., 2014). Recently, hepatic differentiation of hDPSCs was undertaken using cryopreserved dental pulp tissue from teeth 
with disease (Chen et al., 2013). Differentiated cells possessed a polygonal shape and normal karyotype and expressed hepatic metabolic function genes and liverspecific genes. Glycogen storage and urea production results indicated that the differentiated hDPSCs were functionally close to normal HLCs (Chen et al., 2013). Although research into hepatic differentiation of DPSCs is at an early stage, the use of cryopreserved tissue to generate HLCs provides a promising alternative for the treatment of liver diseases.

Diabetes is a degenerative disease in which the autoimmune destruction of pancreatic $\beta$-cells or decreased insulin sensitivity leads to persistent hyperglycaemia. Conventional insulin-based treatments of diabetes have many limitations. Replenishing the lost insulin-producing cells (IPCs), either by islet transplantation or by differentiated stem cells, is a preferred approach for the treatment of diabetes (Bhonde et al., 2013). The potential of DPSCs to differentiate into pancreatic cell lineage resembling islet-like cell aggregates (ICAs) has been explored, with the result that in vitro ICAs glucose-dependently released insulin and C-peptide (Govindasamy et al., 2011). Human DPSCs under appropriate stimuli have also been shown to express genes related to pancreatic $\beta$-cell development and function, including insulin and pancreatic and duodenal homebox-1 (Carnevale et al., 2013). Recently, the physiological relevance of this technology was demonstrated with the generation of islet-like cell clusters (ICCs) from DPSCs and SHEDs (Kanafi et al., 2013a). ICCs from SHEDs reversed hyperglycaemia in experimental diabetic mice and demonstrated that dental pulp can provide an autologous stem cell therapy that could be used for diabetes (Kanafi et al., 2013a).

\subsubsection{IPS cells from dental pulp stem cells}

Recently, Yan et al. (2010b) reprogrammed human DPSCs into iPS cells using the four factors Lin28, Nanog, Oct4 and Sox2 or c-Myc, Klf4, Oct4 and Sox2. The iPS hDPSCs exhibited hES morphology, expressed hES markers and formed embryoid bodies in vitro and teratomas in vivo, containing tissues of all three germ layers. Following on from this, Oda et al. (2010) demonstrated iPS generation of DPSCs by retroviral transduction with Oct3/4, Sox2 and Klf4 without the need for Myc or Lin28. Both these studies showed that DPSCs obtained from human third molars, generally considered clinical waste, are a valuable source for iPS cells. This was further evidenced by the study of Tamaoki et al. (2010), which demonstrated the suitability of DPCs as a source of iPS cell banks. Recently, two non-oncogenic factors, Oct4 and Sox9, were used to generate potential substrates for endothelial progenitor cells (EPCs) from hDPCs (Yoo et al., 2013). The $2 \mathrm{~F}$ hDPC-hiPSCs differentiated into multipotent CD $34^{+}$EPCs capable of differentiating into functional endothelial and smooth muscle cells, with well-documented therapeutic effects in mouse models of hind-limb ischaemia and myocardial infarction (Yoo et al., 2013). This study demonstrates the promising potential of dental pulpderived cells, including DPSCs, for future therapeutic applications utilizing iPS cells.

\section{Concluding remarks}

Human dental pulp offers a fascinating source of adult stem cells. The developmental pathway that generates DPSCs results in a cell type that can contribute to the regeneration of numerous tissue types. Potentially, DPSCs could be easy to bank from teeth that are lost naturally during childhood or removed surgically due to impaction. A large body of literature is building to support the clinical use of DPSCs in dentistry, orthopaedics and other applications.

\section{Acknowledgements}

K.M.S. has received funding from the European Research Council under the European Community's Seventh Framework Programme (Grant No. FP7/2007-2013/ERC Grant Agreement 227845). Funding for research from Calabrodental has been received by the University of Nottingham and K.M.S.

\section{Conflict of interest}

The authors have declared that there is no conflict of interest.

\section{References}

Akkouch A, Zhang Z, Rouabhia M. 2014; Engineering bone tissue using human dental pulp stem cells and an osteogenic collagen-hydroxyapatite-poly (-lactide-co- $\varepsilon$ caprolactone) scaffold. J Biomater Appl 28: 922-936.

Alsanea R, Ravindran S, Fayad MI et al. 2011; Biomimetic pproach to perforation repair using dental pulp stem cells and dentin matrix protein 1. J Endodont 37: 1092-1097.

Annibali S, Bellavia D, Ottolenghi L, et al. 2014; Micro-CT and PET analysis of bone regeneration induced by biodegradable scaffolds as carriers for dental pulp stem cells in a rat model of calvarial 'critical size' defect: preliminary data. J Biomed Mater Res B Appl Biomater 102(4): 815-825.

Apel C, Forlenza OV, de Paula VJR et al. 2009; The neuroprotective effect of dental pulp cells in models of Alzheimer's and Parkinson's disease. J Neural Transm 116: 71-78.

Arthur A, Rychkov G, Shi S et al. 2008; Adult human dental pulp stem cells differentiate toward functionally active neurons under appropriate environmental cues. Stem Cells 26: 1787-1795.
Arthur A, Shi S, Zannettino ACW et al. 2009; Implanted adult human dental pulp stem cells induce endogenous axon guidance. Stem Cells 27: 2229-2237.

Batouli S, Miura M, Brahim J et al. 2003; Comparison of stem-cell-mediated osteogenesis and dentinogenesis. $J$ Dent Res 82: 976-981.

Bhonde RR, Sheshadri P, Sharma S et al. 2013; Making surrogate $\beta$-cells from mesenchymal stromal cells: perspectives and future endeavors. Int J Biochem Cell Biol 46C: 90-102. Bohl KS, Shon J, Rutherford B et al. 1998; Role of synthetic extracellular matrix in 
development of engineered dental pulp. $J$ Biomater Sci Polym Edn 9: 749-764.

Bronckaers A, Hilkens P, Fanton Y, 2013; Angiogenic properties of human dental pulp stem cells. PLoS One 8: e71104.

Carnevale G, Riccio M, Pisciotta A et al. 2013, In vitro differentiation into insulinproducing $\beta$-cells of stem cells isolated from human amniotic fluid and dental pulp. Dig Liver Dis 45: 669-676.

Chen YK, Huang AH, Chan AW et al. 2013; Human dental pulp stem cells derived from cryopreserved dental pulp tissues of vital extracted teeth with disease demonstrate hepatic-like differentiation. J Tissue Eng Regen Med DoI: 10.1002/ term.1763.

Cordeiro MM, Dong Z, Kaneko T et al. 2008; Dental pulp tissue engineering with stem cells from exfoliated deciduous teeth. $J$ Endodont 34: 962-969.

d'Aquino R, Graziano A, Sampaolesi M et al. 2007; Human postnatal dental pulp cells co-differentiate into osteoblasts and endotheliocytes: a pivotal synergy leading to adult bone tissue formation. Cell Death Differ 14: 1162-1171.

Dai W, Hale SL, Kloner RA. 2005; Stem cell transplantation for the treatment of myocardial infarction. Transpl Immunol 15: 91-97.

de Almeida FM, Marques SA, Ramalho BDS et al. 2011; Human dental pulp cells: a new source of cell therapy in a mouse model of compressive spinal cord injury. $J$ Neurotrauma 28: 1939-1949.

Demarco FF, Casagrande L, Zhang Z et al. 2010; Effects of morphogen and scaffold porogen on the differentiation of dental pulp stem cells. $J$ Endodont 36: 1805-1811.

d'Aquino R, De Rosa A, Lanza V et al. 2009; Human mandible bone defect repair by the grafting of dental pulp stem/progenitor cells and collagen sponge biocomplexes. Eur Cell Mater 18: 75-83.

d'Aquino R, Papaccio G, Laino G et al. 2008; Dental pulp stem cells: a promising tool for bone regeneration. Stem Cell Rev 4: 21-26.

El-Backly RM, Massoud AG, El-Badry AM et al. 2008; Regeneration of dentine/ pulp-like tissue using a dental pulp stem cell/poly(lactic-co-glycolic) acid scaffold construct in New Zealand white rabbits. Aus Endodont $J$ 34: 52-67.

Ferro F, Spelat R, Beltrami AP et al. 2012; Isolation and characterization of human dental pulp derived stem cells by using media containing low human serum percentage as clinical grade substitutes for bovine serum (Mezey E, ed.). PLoS One 7: e48945.

Galler KM, D'Souza RN, Hartgerink JD et al. 2011; Scaffolds for dental pulp tissue engineering. Adv Dent Res 23: 333-339.

Gandia C, Armiñan A, García-Verdugo JM et al. 2008; Human dental pulp stem cells improve left ventricular function, induce angiogenesis, and reduce infarct size in rats with acute myocardial infarction. Stem Cells 26: 638-645.

Ge J, Ju Y, Xue Z et al. 2013; Distal C terminus of CaV1.2 channels plays a crucial role in the neural differentiation of dental pulp stem cells. PLoS One 8: e81332.

Gebhardt M, Murray PE, Namerow KN et al. 2009; Cell survival within pulp and periodontal constructs. $J$ Endodont 35: 63-66.
Govindasamy V, Abdullah AN, Ronald VS et al. 2010; Inherent differential propensity of dental pulp stem cells derived from human deciduous and permanent teeth. $J$ Endodont 36: 1504-1515.

Govindasamy V, Ronald VS, Abdullah ANB et al. 2012; Human platelet lysate permits scale-up of dental pulp stromal cells for clinical applications. Cytotherapy 13: 1221-1233.

Govindasamy V, Ronald VS, Abdullah AN et al. 2011; Differentiation of dental pulp stem cells into islet-like aggregates. $J$ Dental Res 90: 646-652.

Gronthos S, Brahim J, Li W et al. 2002; Stem cell properties of human dental pulp stem cells. J Dent Res 81: 531-535.

Gronthos S, Mankani M, Brahim J et al. 2000; Postnatal human dental pulp stem cells (DPSCs) in vitro and in vivo. Proc Natl Acad Sci 97: 13625-13630.

Hilkens P, Gervois P, Fanton Y et al. 2013; Effect of isolation methodology on stem cell properties and multilineage differentiation potential of human dental pulp stem cells. Cell Tissue Res 353: 65-78.

Huang AHC, Chen YK, Lin LM et al. 2008a; Isolation and characterization of dental pulp stem cells from a supernumerary tooth. J Oral Pathol Med 37: 571-574.

Huang AHC, Snyder BR, Cheng PH et al. 2008b; Putative dental pulp-derived stem/stromal cells promote proliferation and differentiation of endogenous neural cells in the hippocampus of mice. Stem Cells 26: 2654-2663.

Huang GT. 2009; Pulp and dentin tissue engineering and regeneration: current progress. Regen Med 4: 697-707.

Huang GTJ. 2011; Dental pulp and dentin tissue engineering and regeneration advancement and challenge. Front Biosci (Elite edn) 3: 788

Huang GTJ, Gronthos S, Shi S. 2009a; Mesenchymal stem cells derived from dental tissues vs. those from other sources: their biology and role in regenerative medicine. J Dent Res 88: 792-806.

Huang GTJ, Sonoyama W, Chen J et al. 2006; In vitro characterization of human dental pulp cells: various isolation methods and culturing environments. Cell Tissue Res 324: 225-236.

Huang GTJ, Yamaza T, Shea LD et al. 2009b; Stem/progenitor cell-mediated de novo regeneration of dental pulp with newly deposited continuous layer of dentin in an in vivo model. Tissue Eng A 16: 605-615.

Huang GT, Yamaza T, Shea LD et al. 2010; Stem/progenitor cell-mediated de novo regeneration of dental pulp with newly deposited continuous layer of dentin in an in vivo model. Tissue Eng Part A 16: 605-615.

Hung CN, Mar K, Chang HC et al. 2011; A comparison between adipose tissue and dental pulp as sources of MSCs for tooth regeneration. Biomaterials 32: 6995-7005.

Ikeda E, Morita R, Nakao K et al. 2009; Fully functional bioengineered tooth replacement as an organ replacement therapy. Proc Nat Acad Sci U S A 106: 13475-13480.

Ikeda H, Sumita Y, Ikeda M et al. 2010; Engineering bone formation from human dental pulp- and periodontal ligamentderived cells. Ann Biomed Eng 39: 26-34.

Iohara $\mathrm{K}$, Imabayashi $\mathrm{K}$, Ishizaka $\mathrm{R}$ et al. 2011; Complete pulp regeneration after pulpectomy by transplantation of $\mathrm{CD} 105^{+}$ stem cells with stromal cell-derived factor-1. Tissue Eng A 17: 1911-1920.

Iohara K, Murakami M, Takeuchi $\mathrm{N}$ et al. 2013; A novel combinatorial therapy with pulp stem cells and granulocyte colonystimulating factor for total pulp regeneration. Stem Cells Transl Med 2: 521-533.

Iohara K, Nakashima M, Ito M et al. 2004; Dentin regeneration by dental pulp stem cell therapy with recombinant human bone morphogenetic protein 2. J Dent Res 83: 590-595.

Iohara K, Zheng L, Ito M et al. 2009; Regeneration of dental pulp after pulpotomy by transplantation of $\mathrm{CD} 31^{-} / \mathrm{CD} 146^{-}$side population cells from a canine tooth. Regen Med 4: 377-385.

Iohara K, Zheng L, Ito M et al. 2006; Side population cells isolated from porcine dental pulp tissue with self-renewal and multipotency for dentinogenesis, chondrogenesis, adipogenesis, and neurogenesis. Stem Cells 24: 2493-2503.

Iohara $\mathrm{K}$, Zheng L, Wake $\mathrm{H}$ et al. 2008; A novel stem cell source for vasculogenesis in ischemia: subfraction of side population cells from dental pulp. Stem Cells 26: 2408-2418.

Ishizaka R, Iohara K, Murakami M et al. 2012; Regeneration of dental pulp following pulpectomy by fractionated stem/progenitor cells from bone marrow and adipose tissue. Biomaterials 33: 2109-2118.

Isner JM, Asahara T, Takahashi T et al. 1999; Ischemia- and cytokine-induced mobilization of bone marrow-derived endothelial progenitor cells for neovascularization. Nat Med 5: 434-438.

Ishkitiev N, Yaegaki K, Imai T et al. 2012 High-purity hepatic lineage differentiated from dental pulp stem cells in serum-free medium. $J$ Endodont 38: 475-480.

Janebodin $\mathrm{K}$, Horst OV, Ieronimakis $\mathrm{N}$ et al. 2011; Isolation and characterization of neural crest-derived stem cells from dental pulp of neonatal mice. PLoS One 6 : e27526.

Kanafi MM, Rajeshwari YB, Gupta $S$ et al. 2013a; Transplantation of islet-like cell clusters derived from human dental pulp stem cells restores normoglycemia in diabetic mice. Cytotherapy 15: 1228-1236.

Kanafi MM, Ramesh A, Gupta PK et al. 2013b; Dental pulp stem cells immobilized in alginate microspheres for applications in bone tissue engineering. Int Endod J DOI: 10.1111/iej.12205.

Karaoz E, Demircan PC, Sağlam Ö et al. 2011; Human dental pulp stem cells demonstrate better neural and epithelial stem cell properties than bone marrow-derived mesenchymal stem cells. Histochem Cell Biol 136: 455-473.

Kawashima N. 2012; Characterisation of dental pulp stem cells: a new horizon for tissue regeneration? Arch Oral Biol 57: 1439-1458.

Kerkis I, Caplan AI. 2012; Stem cells in dental pulp of deciduous teeth. Tissue Eng $B$ Rev 18: 129-138.

Kerkis I, Kerkis A, Dozortsev D et al. 2006; Isolation and characterization of a population of immature dental pulp stem cells expressing OCT-4 and other embryonic stem cell markers. Cells Tissues Organs 184: 105-116.

Kim BC, Bae H, Kwon IK et al. 2012 Osteoblastic/cementoblastic and neural differentiation of dental stem cells and 
their applications to tissue engineering and regenerative medicine. Tissue Eng $B$ Rev 18: 235-244.

Kim NR, Lee DH, Chung PH et al. 2009; Distinct differentiation properties of human dental pulp cells on collagen, gelatin, and chitosan scaffolds. Oral Surg Oral Med Oral Pathol Oral Radiol Endod 108: e94-100

Király M, Kádár K, Horváthy DB et al. 2011; Integration of neuronally predifferentiated human dental pulp stem cells into rat brain in vivo. Neurochemistry 59: 371-381.

Kodonas K, Gogos C, Papadimitriou S et al. 2012; Experimental formation of dentinlike structure in the root canal implant model using cryopreserved swine dental pulp progenitor cells. $J$ Endodont $\mathbf{3 8}$ 913-919.

Kolind K, Kraft D, Boggild T et al. 2014; Control of proliferation and osteogenic differentiation of human dental-pulp-derived stem cells by distinct surface structures. Acta Biomater 10: 641-650.

Laino G, Carinci F, Graziano A et al. 2006; In vitro bone production using stem cells derived from human dental pulp. $J$ Craniofac Surg 17: 511

Laino G, d'Aquino R, Graziano A et al. 2005; A new population of human adult dental pulp stem cells: a useful source of living autologous fibrous bone tissue (LAB). $J$ Bone Miner Res 20: 1394-1402.

Lee CH, Hajibendeh J, Suzuki T et al. 2014; 3D printed multiphase scaffolds for regeneration of periodontium complex. Tissue Eng Part A 20(7-8): 1342-1351.

Lee SY, Chiang PC, Tsai YH et al. 2010; Effects of cryopreservation of intact teeth on the isolated dental pulp stem cells. $J$ Endodont 36: 1336-1340.

Leong WK, Henshall TL, Arthur A, et al. 2012; Human adult dental pulp stem cells enhance poststroke functional recovery through non-neural peplacement mechanisms. Stem Cells Transl Med 1: 177-187.

Lindroos B, Mäenpää K, Ylikomi T et al. 2008; Characterisation of human dental stem cells and buccal mucosa fibroblasts. Biochem Biophys Res Commun 368: 329-335.

Lindvall O, Kokaia Z, Martinez-Serrano A. 2004; Stem cell therapy for human neurodegenerative disorders - how to make it work. Nat Med 10: S42-50.

Liu H, Gronthos S, Shi S. 2006; Dental pulp stem cells. Methods Enzymol 419: 99-113.

Lizier NF, Kerkis A, Gomes CM et al. 2012; Scaling-up of dental pulp stem cells isolated from multiple niches (Pruszak J, ed.). PLoS One 7: e39885.

Mangano C, De Rosa A, Desiderio V et al. 2010; The osteoblastic differentiation of dental pulp stem cells and bone formation on different titanium surface textures. Biomaterials 31: 3543-3551.

Maraldi T, Riccio M, Pisciotta A et al. 2013; Human amniotic fluid-derived and dental pulp-derived stem cells seeded into collagen scaffold repair critical-size bone defects promoting vascularization. Stem Cell Res Ther 4: 53.

Martens W, Bronckaers A, Politis C et al. 2013; Dental stem cells and their promising role in neural regeneration: an update. Clin Oral Inves 17: 1969-1983.

Mead B, Logan A, Berry M et al. 2013; Intravitreally transplanted dental pulp stem cells promote neuroprotection and axon regeneration of retinal ganglion cells after optic nerve injury. Invest Ophthalmol Vis Sci 54: 7544-7556.

Mitsiadis TA, Feki A, Papaccio G et al. 2011; Dental pulp stem cells, niches, and Notch signaling in tooth injury. Adv Dent Res 23: 275-279.

Mooney DJ, Powell C, Piana J et al. 1996; Engineering dental pulp-like tissue in vitro. Biotechnol Progr 12: 865-868.

Nakashima M, Akamine A. 2005; The application of tissue engineering to regeneration of pulp and dentin in endodontics. $J$ Endodont 31: 711-718.

Nakashima M, Iohara K. 2011; Regeneration of dental pulp by stem cells. Adv Denta Res 23: 313-319.

Nakashima M, Iohara K, Sugiyama M. 2009; Human dental pulp stem cells with highly angiogenic and neurogenic potential for possible use in pulp regeneration. Cytokine Growth Factor Rev 20: 435-440.

Nam S, Won JE, Kim CH et al. 2011; Odontogenic differentiation of human dental pulp stem cells stimulated by the calcium phosphate porous granules. $J$ Tissue Eng 2: 812547.

Nosrat IV, Smith CA, Mullally P et al. 2004; Dental pulp cells provide neurotrophic support for dopaminergic neurons and differentiate into neurons in vitro; implications for tissue engineering and repair in the nervous system. Eur $J$ Neurosci 19 2388-2398.

Nosrat IV, Widenfalk J, Olson L et al. 2001; Dental pulp cells produce neurotrophic factors, interact with trigeminal neurons in vitro, and rescue motoneurons after spinal cord injury. Dev Biol 238: 120-132.

Oda Y, Yoshimura Y, Ohnishi H et al. 2010; Induction of pluripotent stem cells from human third molar mesenchymal stroma cells. J Biol Chem 285: 29270-29278.

Ohazama A, Modino SAC, Miletich I et al. 2004; Stem cell-based tissue engineering of murine teeth. $J$ Dent Res $\mathbf{8 3}$ 518-522.

Okamoto Y, Sonoyama W, Ono M et al. 2009, Simvastatin induces the odontogenic differentiation of human dental pulp stem cells in vitro and in vivo. $J$ Endodont 35 367-372.

Patil R, Kumar BM, Lee WJ et al. 2014 Multilineage potential and proteomic profiling of human dental stem cells derived from a single donor. Exp Cell Res 20: 92-107.

Prescott RS, Alsanea R, Fayad MI et al. 2008 In vivo generation of dental pulp-like tissue by using dental pulp stem cells, a collagen scaffold, and dentin matrix protein 1 after subcutaneous transplantation in mice. $J$ Endodont 34: 421-426.

Qu T, Liu X. 2013; Nano-structured gelatin/bioactive glass hybrid scaffolds for the enhancement of odontogenic differentiation of human dental pulp stem cells. J Mater Chem B Mater Biol Med 1: 4764-4772.

Ravindran S, Zhang Y, Huang CC et al. 2014; Odontogenic induction of dental stem cells by extracellular matrix-inspired three-dimensional scaffold. Tissue Eng $A$ 20: 92-102.

Sakai K, Yamamoto A, Matsubara K et al. 2012; Human dental pulp-derived stem cells promote locomotor recovery after complete transection of the rat spinal cord by multiple neuro-regenerative mechanisms. J Clin Invest 122: 80.

Sasaki R, Aoki S, Yamato M. 2011; PLGA artificial nerve conduits with dental pulp cells promote facial nerve regeneration. $J$ Tissue Eng Regen Med 5: 823-830.

Sasaki R, Aoki S, Yamato M et al. 2008a; Neurosphere generation from dental pulp of adult rat incisor. Eur $J$ Neurosci 27: 538-548.

Sasaki R, Aoki S, Yamato M et al. 2008b; Tubulation with dental pulp cells promotes facial nerve regeneration in rats. Tissue Eng A 14: 1141-1147.

Shi S, Gronthos S. 2003; Perivascular niche of postnatal mesenchymal stem cells in human bone marrow and dental pulp. $J$ Bone Miner Res 18: 696-704.

Shi S, Bartold PM, Miura M et al. 2005; The efficacy of mesenchymal stem cells to regenerate and repair dental structures. Orthodont Craniofac Res 8: 191-199.

Sloan AJ, Smith AJ. 2007; Stem cells and the dental pulp: potential roles in dentine regeneration and repair. Oral Dis 13: 151-157.

Sonoyama W, Liu Y, Fang D et al. 2006 Mesenchymal stem cell-mediated functional tooth regeneration in swine. PLOS One 1: e79.

Srisuwan T, Tilkorn DJ, Al-Benna S et al. 2012a; Revascularization and tissue regeneration of an empty root canal space is en hanced by a direct blood supply and stem cells. Dent Traumatol 29: 84-91.

Srisuwan T, Tilkorn DJ, Al-Benna S et al. 2012b; Survival of rat functional dental pulp cells in vascularized tissue engineering chambers. Tissue Cell 44: 111-121.

Sugiyama M, Iohara K, Wakita H et al. 2011; Dental pulp-derived CD31 ${ }^{-} / \mathrm{CD}_{146}{ }^{-}$side population stem/progenitor cells enhance recovery of focal cerebral ischemia in rats. Tissue Eng A 17: 1303-1311.

Sun HH, Jin T, Yu Q et al. 2011; Biological approaches toward dental pulp regeneration by tissue engineering. $J$ Tissue Eng Regen Med 5: e1-16.

Takeyasu M, Nozaki T, Daito M. 2006; Differentiation of dental pulp stem cells into a neural lineage. Pediatr Dent $J$ 16: 154-162.

Tamaoki N, Takahashi K, Tanaka $\mathrm{T}$ et al. 2010; Dental pulp cells for induced pluripotent stem cell banking. $J$ Dent Res 89: 773-778.

Tucker A, Sharpe P. 2004; The cutting-edge of mammalian development; how the embryo makes teeth. Nature Rev Genet 5: 499-508.

Volponi AA, Pang Y, Sharpe PT. 2010; Stem cell-based biological tooth repair and regeneration. Trends Cell Biol 20: 715-722.

Volponi A, Kawasaki M, Sharpe PT. 2013; Adult human gingival epithelial cells as a source for whole-tooth bioengineering. $J$ Dent Res 92: 329-334.

Wang J, Liu X, Jin X et al. 2010; The odontogenic differentiation of human dental pulp stem cells on nanofibrous poly(L-lactic acid) scaffolds in vitro and in vivo. Acta Biomater 6: 3856-3863.

Wang J, Ma H, Jin X et al. 2011; The effect of scaffold architecture on odontogenic differentiation of human dental pulp stem cells. Biomaterials 32: 7822-7830.

Wollert KC, Drexler H. 2005; Mesenchyma stem cells for myocardial infarction: prom ises and pitfalls. Circulation 112: 151-153.

Woods EJ, Perry BC, Hockema JJ et al. 2009 Optimized cryopreservation method for 
human dental pulp-derived stem cells and their tissues of origin for banking and clinical use. Cryobiology 59: 150-157.

Yamada Y, Ito K, Nakamura S et al. 2011; Promising cell-based therapy for bone regeneration using stem cells from deciduous teeth, dental pulp, and bone marrow. Cell Transpl 20: 1003-1013.

Yamada Y, Nakamura S, Ito K et al. 2010; A feasibility of useful cell-based therapy by bone regeneration with deciduous tooth stem cells, dental pulp stem cells, or bone-marrow-derived mesenchymal stem cells for clinical study using tissue engineering technology. Tissue Eng A 16: 1891-1900.

Yamamoto A, Sakai K, Matsubara $\mathrm{K}$ et al. 2014; Multifaceted neuro-regenerative activities of human dental pulp stem cells for functional recovery after spinal cord injury. Neurosci Res 78: 16-20.

Yan M, Yu Y, Zhang G et al. 2010a; A journey from dental pulp stem cells to a bio-tooth. Stem Cell Rev 7: 161-171.
Yan X, Qin H, Qu C et al. 2010b; iPS cells reprogrammed from human mesenchymallike stem/progenitor cells of dental tissue origin. Stem Cells Dev 19: 469-480.

Yang KL, Chen MF, Liao CH et al. 2012; A simple and efficient method for generating Nurr1-positive neuronal stem cells from human wisdom teeth (tNSC) and the potential of tNSC for stroke therapy. Cytotherapy 11: 606-617.

Yang X, van der Kraan PM, Bian Z et al. 2009a; Mineralized tissue formation by BMP2-transfected pulp stem cells. $J$ Dent Res 88: 1020-1025.

Yang X, Walboomers XF, van den Beucken JJJP et al. 2009b; Hard tissue formation of STRO-1-selected rat dental pulp stem cells in vivo. Tissue Eng A 15: 367-375.

Yoo CH, Na HJ, Lee DS et al. 2013; Endothelial progenitor cells from human dental pulp-derived iPS cells as a therapeutic target for ischemic vascular diseases. Biomaterials 34: 8149-8160.
Young F, Sloan A, Song B et al. 2013; Dental pulp stem cells and their potential roles in central nervous system regeneration and repair. J Neurosci Res 91: 1383-1393.

Yu J, Wang Y, Deng Z et al. 2012; Odontogenic capability: bone marrow stromal stem cells versus dental pulp stem cells. Biol Cell 99: 465-474.

Zhang W, Abukawa H, Troulis MJ et al. 2009; Tissue engineered hybrid tooth-bone constructs. Methods 47: 122-128.

Zhang W, Walboomers XF, Shi S et al. 2006; Multilineage differentiation potential of stem cells derived from human dental pulp after cryopreservation. Tissue Eng 12: 2813-2823.

Zhang YD, Zhi C, Yi Qiang S et al. 2005; Making a tooth: growth factors, transcription factors, and stem cells. Cell Res 15: 301-316.

Zheng Y, Wang XY, Wang YM et al. 2012; Dentin regeneration using deciduous pulp stem/progenitor cells. J Dent Res 91: 676-682. 